\title{
Design of Cam Mechanism with Negative Radius Roller-Follower
}

\author{
Jung-Fa Hsieh ${ }^{1, a}$ and Wen-Chang Hsieh ${ }^{2}$ \\ ${ }^{1}$ Department of Mechanical Engineering, Far East University, Tainan, Taiwan \\ ${ }^{2}$ Department of Business Administration, Yunlin University of Science \& Technology, Yunlin, Taiwan
}

\begin{abstract}
Cam mechanisms with a negative radius roller-follower allow the design of simple machines capable of exerting remarkably high forces. Furthermore, the concave-convex nature of the contact surface between the cam and the follower results in a low contact stress and the ready formation of a lubricating layer. This paper presents a simple method for the design and analysis of a cam mechanism with a negative radius roller-follower. In the proposed approach, conjugate surface theory is employed to derive a kinematic model of the cam mechanism. Analytical expressions for the pressure angle and principal curvatures of the cam profile are then derived. The validity of the proposed design methodology is demonstrated by machining a cam mechanism having a negative radius rollerfollower with a radius of $100 \mathrm{~mm}$.
\end{abstract}

\section{Introduction}

Cam elements have many advantageous features for mechanical transmission systems, including simplicity, reliability, repeatability and a low running noise. Contact pairs comprising a convex cylinder and a concave cylinder have a large contact area, and hence a low contact stress [1]. Thus, cam profiles generally have a convex face, while follower profiles have either a convex face or a flat face. In order to distinguish conventional construct, followers with a concave face are referred to as negative radius followers. Cams with negative radius roller-followers use the inner rings of commercial roller bearings as roller-followers. the use of such mechanisms is particularly valuable in applications where space is at a premium and very high forces are involved. Carra et al. [2] presented a design nomograph and synthesis procedure for finding the suitable combination of parameters to satisfy the specified motion law without under-cutting. Wu et al. [3] compared the contact stress of two disc cam mechanisms with a concave-faced follower and a flat-faced follower, respectively. The results showed that the cam mechanism with a concavefaced follower produced a lower maximum contact stress than that with a common flat-faced follower. Moreover, it was shown that the negative radius roller-follower caused the sliding friction between the cam and the follower to be converted to rolling friction. However, the literature lacks a systematic investigation into the design of cam mechanisms with negative radius roller-followers. Accordingly, this study presents a simple yet robust methodology for the design and analysis of such mechanisms. The proposed methodology comprises four steps, namely (1) establishing a kinematic model of the cam mechanism, (2) designing the cam profile, and (3) analyzing the pressure angle and principal curvatures of the cam.

\section{Surface geometry}

In order to analyze the contact stress in a cam mechanism, it is first necessary to determine the profile of the cam. As discussed above, cams with negative radius rollerfollowers use the inner rings of commercial roller bearings as followers. In other words, the cam fits within an inner ring. In synthesizing the cam profile, the cam mechanism can be represented using the simplified model shown in Fig. 1. As shown, the cam rotates with an angular speed $w_{1}$ in the clockwise direction about its center point, $0_{0}$. As the cam rotates, the follower rises to a total lift height $\mathrm{h}$ over a specified rise angle, remains in a high dwell position over the high dwell angle, falls through a distance $\mathrm{h}$ over the return angle, and then remains in a low dwell position over the low dwell angle.

To determine the cam profile using conjugate theory, it is necessary to label all of the coordinate frames in the system, starting with the cam (marked as "0" in Fig. 1) and ending with the translating follower (marked as "2" in Fig.1). Note that parameter $b_{2}$ is defined as $b_{2}=r_{\mathrm{b}}+s_{2}(1)$, where $r_{\mathrm{b}}$ is the base circle radius of the cam and $s_{2}$ is the translating follower displacement.

The configuration of frame $(x y z)_{2}$ with respect to frame $(x y z)_{0}$ is given by

${ }^{0} \mathbf{T}_{2}={ }^{0} \mathbf{T}_{1}{ }^{1} \mathbf{T}_{2}=\left[\begin{array}{ccc}C \theta_{1} & -S \theta_{1} & -b_{2} S \theta_{1} \\ S \theta_{1} & C \theta_{1} & b_{2} C \theta_{1} \\ 0 & 0 & 1\end{array}\right]$

${ }^{a}$ Corresponding author: seznof@cc.feu.edu.tw 


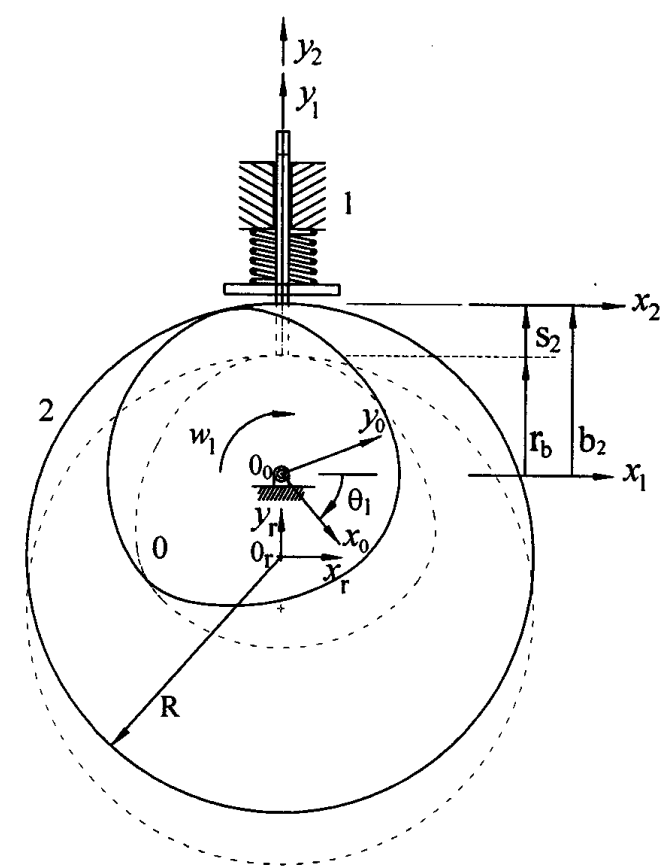

Fig.1. Simplified representation of cam and follower mechanism for synthesis of cam profile.

To express the surface of the roller in terms of frame $(x y z)_{0}$, it is first necessary to establish the frame of the roller-follower, namely $(x y z)_{\mathrm{r}}$. The configuration of frame $(x y z)_{\mathrm{r}}$ with respect to frame $(x y z)_{2}$ is given by the matrix

$$
{ }^{2} \mathbf{T}_{r}=\left[\begin{array}{ccc}
1 & 0 & 0 \\
0 & 1 & -R \\
0 & 0 & 1
\end{array}\right]
$$

where $R$ is the radius of the roller.

The surface equation, ${ }^{\mathrm{r}} \boldsymbol{S}$, and unit outward normal, ${ }^{\mathrm{r}} \mathbf{n}$, of the roller can be expressed with respect to frame $(x y z)_{\mathrm{r}}$ as follows:

$$
\begin{aligned}
& { }^{r} S=\left[\begin{array}{lll}
R C \theta & R S \theta & 1
\end{array}\right]^{T} \\
& { }^{r} n=\left[\begin{array}{lll}
R C \theta & R S \theta & 0
\end{array}\right]^{T}
\end{aligned}
$$

where $\theta$ is the polar angle.

In accordance with the principles of homogeneous coordinate transformation, the cam surface equation, ${ }^{0} \boldsymbol{S}$, can be expressed as

$$
{ }^{0} \mathbf{S}={ }^{0} \mathbf{T}_{2}{ }^{2} \mathbf{T}_{r}{ }^{r} \mathbf{S}=\left[\begin{array}{c}
R C\left(\theta+\theta_{1}\right)+\left(R-b_{2}\right) S \theta_{1} \\
R S\left(\theta+\theta_{1}\right)+\left(b_{2}-R\right) C \theta_{1} \\
1
\end{array}\right]
$$

In addition, the normal vector, ${ }^{0} \mathbf{n}$, of the cam can be expressed as

$$
{ }^{0} \mathbf{n}={ }^{0} \mathbf{T}_{2}{ }^{2} \mathbf{T}_{r}{ }^{r} \mathbf{n}=\left[\begin{array}{lll}
C\left(\theta+\theta_{1}\right) & S\left(\theta+\theta_{1}\right) & 0
\end{array}\right]^{T}
$$

Once the input-output relation of the cam mechanism has been defined, the conjugate points and cam profiles can be determined via the formulation

$$
{ }^{0} \mathbf{n}^{T} \bullet \frac{d^{0} \mathbf{S}}{d t}=0
$$

where ${ }^{0} \mathbf{n}$ and ${ }^{0} \mathbf{S}$ are the unit outward normal and surface equation of the cam profile with respect to frame $(x y z)_{0}$, respectively. The conjugate point (denoted as $\bar{\theta}$ ) is given by

$$
\bar{\theta}=-\tan ^{-1}\left(\frac{\bar{F}}{\bar{E}}\right)
$$

where $\bar{E}$ is defined as $\bar{E}=b_{2}-R$ and $\bar{F}$ is defined as $\bar{F}=\frac{d b_{2}}{d \theta_{1}}$.

Substituting Eq.(8) into Eq.(5), the profile of the cam, ${ }^{0} \mathrm{~S}$, can be obtained as

$$
{ }^{0} \mathbf{S}=\left[\begin{array}{c}
R C\left(\bar{\theta}+\theta_{1}\right)+\left(R-b_{2}\right) S \theta_{1} \\
R S\left(\bar{\theta}+\theta_{1}\right)+\left(b_{2}-R\right) C \theta_{1} \\
1
\end{array}\right]
$$

\section{Pressure angle}

As shown in Fig. 2, the pressure angle is defined as the angle between the direction of motion (i.e., velocity) of the follower and the direction of the force transmission axis [5]. The pressure angle at the contact point $p$ between the cam and the roller is given by

$\tan \psi=\frac{\left\|{ }^{1} \mathbf{n}_{p} \times{ }^{1} \mathbf{v}_{p}\right\|}{\left|{ }^{1} \mathbf{n}_{p} \bullet \mathbf{v}_{p}\right|}$

where the unit normal vector of the contact point, ${ }^{1} \mathbf{n}_{\mathrm{p}}$, and the direction of velocity of the contact point, ${ }^{1} \mathbf{v}_{\mathrm{p}}$, are both defined with respect to the fixed frame $(x y z)_{1}$.



Fig.2 Pressure angle of cam mechanism during the rise of the follower

The unit normal vector of the contact point on the roller-follower surface with respect to frame $(x y z)_{1}$ is given by

$$
{ }^{1} \mathbf{n}_{p}={ }^{1} \mathbf{T}_{2}{ }^{2} \mathbf{T}_{r}{ }^{r} \mathbf{n}_{p}=\left[\begin{array}{lll}
C \bar{\theta} & S \bar{\theta} & 0
\end{array}\right]^{T}
$$

Meanwhile, the location of the contact point, with respect to frame $(x y z)_{1}$. is given by 
${ }^{1} \mathbf{S}_{p}={ }^{1} \mathbf{T}_{2}{ }^{2} \mathbf{T}_{r}{ }_{r}^{r} \mathbf{S}_{p}=\left[\begin{array}{lll}R C \bar{\theta} & R S \bar{\theta}-R+b_{2} & 1\end{array}\right]^{T}$

Since the roller-follower translates along the $y_{1}$ axis direction, the direction of velocity of the contact point on the roller-follower surface with respect to frame $(x y z)_{1}$. can be expressed as

${ }^{1} \mathbf{v}_{p}=\frac{d^{1} \mathbf{S}}{d t}=\left[\begin{array}{lll}0 & \frac{d b_{2}}{d t} & 0\end{array}\right]^{T}$

Substituting Eqs.(11) and (13) into Eq.(10), the pressure angle, $\psi$, can be derived as

$\tan \psi=\frac{d b_{2}}{d \theta_{1}} /\left(R-b_{2}\right)=\frac{d b_{2}}{d \theta_{1}} / R-\left(r_{b}+s_{2}\right)$

Equation (14) shows that an increase of the base circle radius $r_{\mathrm{b}}$ leads to a lower pressure angle $\psi$. Notably, a higher maximum velocity causes an undesirable increase of the contact pressure angle. However, for negative radius (concave-faced) follower cams, the adverse effects of a higher pressure angle are generally insignificant.

\section{Analysis of curvature radius}

The curvature radius of the cam must be analyzed in order to prevent the occurrence of singular points. Note that the radius of curvature of the cam cannot be greater than the radius of the follower since unintended multiplepoint contacts will otherwise occur [6]. According to basic differential geometry principles, the principal curvatures of the cam profile can be evaluated as follows:

$K_{1}, K_{2}=H \pm \sqrt{H^{2}-K}$

where $K_{1}$ and $K_{2}$ are the principal curvatures, and $K$ and $H$ are defined respectively as

$K=\frac{L N-M^{2}}{E G-F^{2}}$ and $H=\frac{2 F M-E N-G L}{2\left(E G-F^{2}\right)}$

where $\quad L={ }^{0} n \bullet \frac{\partial^{20} S}{\partial^{2} u} \quad, \quad M={ }^{0} n \bullet \frac{\partial^{20} S}{\partial u \partial \theta_{1}}$, $N={ }^{0} n \bullet \frac{\partial^{20} S}{\partial^{2} \theta_{1}}, E=\frac{\partial^{0} S}{\partial u} \bullet \frac{\partial^{0} S}{\partial u} \quad, \quad F=\frac{\partial^{0} S}{\partial u} \bullet \frac{\partial^{0} S}{\partial \theta_{1}}$, and $G=\frac{\partial^{0} S}{\partial \theta_{1}} \bullet \frac{\partial^{0} S}{\partial \theta_{1}}$

The present study considers the case of a planar cam. Hence, one of the principle curvatures is equal to zero. Moreover, the curvature radius, $\rho$, of the cam profile is equal to the inverse of the principal curvature

\section{Implementation}

To validate the design methodology presented in Sections $2 \sim 4$, a cam mechanism was designed with a negative radius roller-follower having a radius of $100 \mathrm{~mm}$. The motions of the cam and follower were defined by a modified sine motion curve. The follower motion was specified as follows: $20 \mathrm{~mm}$ rise for cam rotation from 0 to $150^{\circ}$, dwell for cam rotation to $180^{\circ}, 20 \mathrm{~mm}$ fall for cam rotation to $330^{\circ}$, and dwell for the remaining $30^{\circ}$. The radius base circle was specified as $40 \mathrm{~mm}$.

Figure 3 shows the simulation results obtained for the cam profile. Figure 4 shows the variation of the pressure angle at the contact point as the cam rotates. Figure 5 shows the curvature radius of the cam profile. Figure 6 presents a photograph of the machined cam. Note that the process of generating the $\mathrm{NC}$ equations required to produce the driving cam on a 3 -axis machine tool is described in [7]. Note also that the three holes lying along the $\mathrm{x}_{0}$ axis of the cam are the workpiece origin (center hole) and clamping holes, respectively. It is seen that a good qualitative agreement exists between the machined profile and the designed profile (see Fig. 3). Thus, the feasibility of the proposed methodology is confirmed.

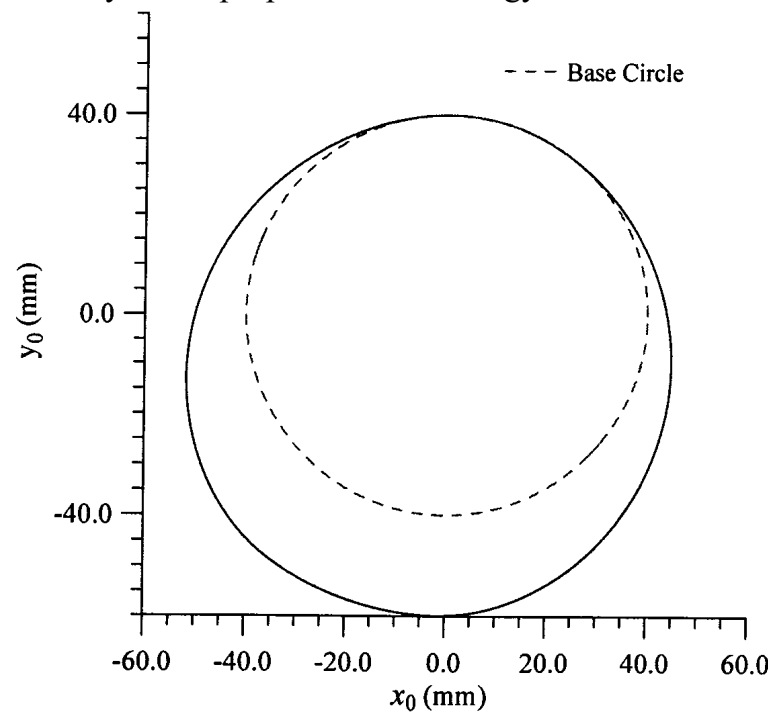

Fig.3 Simulated profile of cam with negative radius rollerfollower

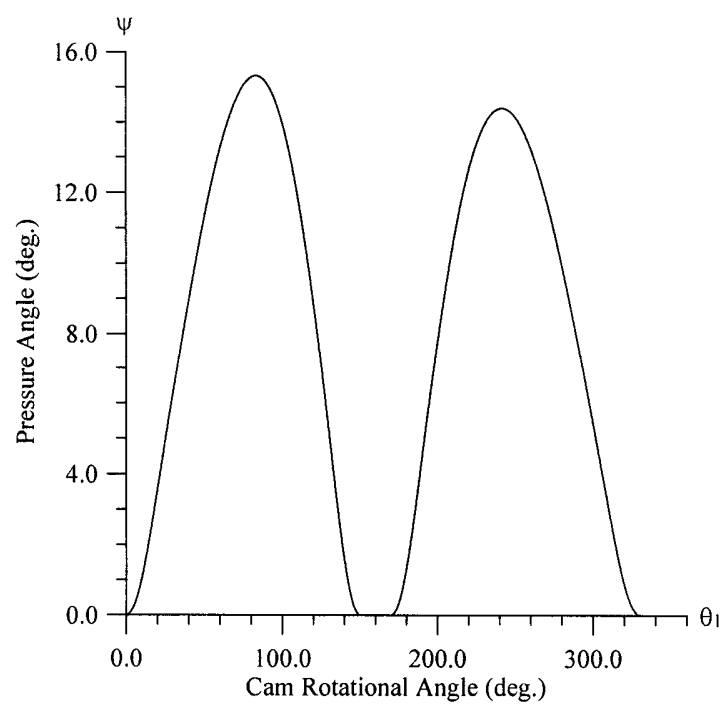

Fig.4 Variation of pressure angle with cam rotational angle 


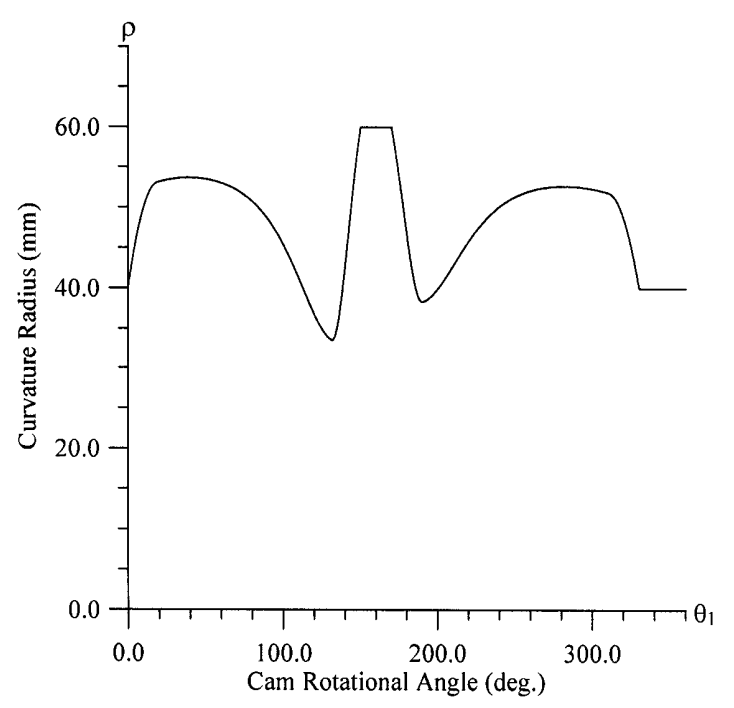

Fig.5 Variation of curvature radius with cam rotational angle

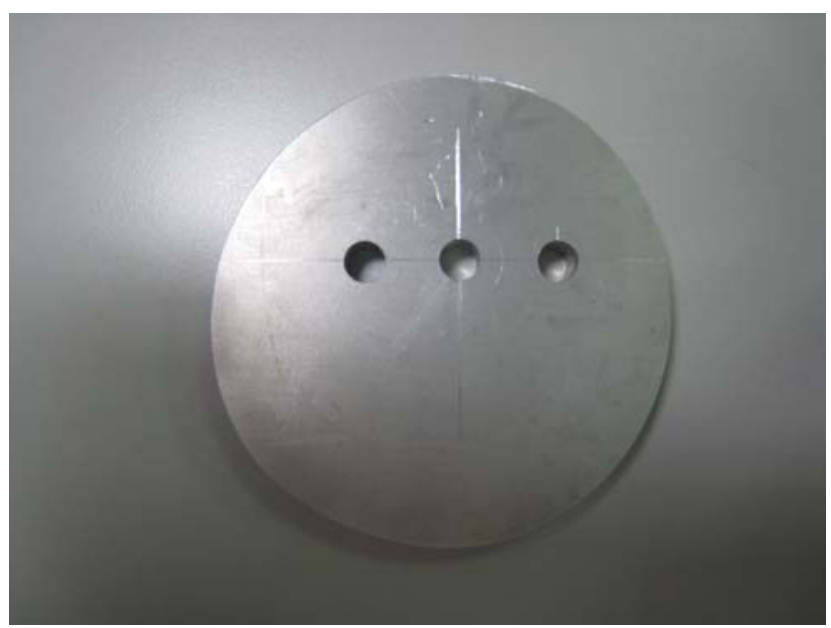

Fig.6 Photograph of machined cam

\section{Conclusions}

This paper has presented an integrated methodology for the design of a cam mechanism with a negative radius roller-follower. A kinematic model of the cam mechanism has been derived utilizing the homogeneous coordinate transformation method and conjugate surface theory. In addition, analytical expressions have been presented for the pressure angle and principal curvatures of the cam profile. The feasibility of the proposed approach has been demonstrated both numerically and experimentally.

\section{References}

1. Collins,J. A., Mechanical design of machine elements and Machines, New York: John Wiley \& Sons Press (2003)

2. Carra, S., Garziera, R. and Pellegrini, M., Synthesis of cams with negative radius follower and evaluation of the pressure angle, Mechanism and Machine Theory, 39, 1017-1032 (2004)
3. Wu, L.-I., Liu, C-H and Chen, T.-W., Disc cam mechanisms with concave-faced followers, Proc. Instn.Mech, Part C: J. Mechanical Engineering Science, 223, 1443-1448 (2009)

4. McCarthy, J. Michael, Geometric Design of Linkage, New York: Springer-Verlag (2000)

5. Gonzalez-Palacios, M.A. and Angles, J., Cam Synthesis, Springer (1993)

6. Jones, J. Rees, Mechanisms-cam curvature and interference, Engineering, 218, 460-463 (1978)

7. Hsieh, Jung-Fa, Application of homogenous transformation matrix to the design and machining of a Geneva mechanism with curved slots, Proc. Instn. Mech. Engrs, Part C: J. Mechanical Engineering Science, 221, 1435-1443 (2007) 